\title{
Tomographic imaging with single atom sensitivity using aberration-corrected STEM
}

K. van Benthem, A.R. Lupini, Y. Peng, S.J. Pennycook

Oak Ridge National Laboratory, Condensed Matter Science Division, Oak Ridge, TN 37831-6031, USA

The distribution of single dopant atoms can dramatically alter the properties of semiconductors and catalysts, which are arguably the two most important classes of modern materials. The detectability and lateral localization of such single atoms has been greatly improved by the recent development of aberration correction in scanning transmission electron microscopy (STEM) [1], which enables the formation of electron probes with diameters that can be as small as 0.06-0.08 nm [2]. We take advantage of a previously unappreciated effect of aberration correction, the increase in depth resolution. By consecutively focusing the electron probe into different planes of the specimen, a slice-by-slice image of the three-dimensional structure is available, which is realized by recording through-focal series (see Figure 1). The recorded image stacks can then be used for 3-D reconstructions.

We show how the improved vertical resolution along with sub-Ångstrom lateral resolution enables the direct and unequivocal three-dimensional location of single $\mathrm{Hf}$ atoms inside a semiconductor device structure. The $\mathrm{Hf}$ atoms are visible in a narrow $\mathrm{SiO}_{2}$ layer located between a single-crystalline $\mathrm{Si}$ wafer and a polycrystalline $\mathrm{HfO}_{2}$ dielectric film, but are not in contact with the Si substrate (see Figure 2a). All individual $\mathrm{Hf}$ atoms remain at least $0.3 \mathrm{~nm}$ away from the $\mathrm{Si} / \mathrm{SiO}_{2}$ interface. This atom by atom characterization of semiconductor interfaces in all three dimensions is a critical capability for the prediction of gate-dielectric properties and the design of new semiconductor devices.

In a second example, we show three-dimensional data sets obtained from $\mathrm{Au}$ nano-crystals on a carbon support film. Isolated $\mathrm{Au}$ atoms beside some $\mathrm{Au}$ clusters indicate the vertical position of the support film (cf. Figure $2 b$ ). The threedimensional shape of the Au clusters can be determined by a combination with image simulations [3].

\section{References:}

[1] P.E. Batson, N. Dellby, O.L. Krivanek, Nature 418, 617-620 (2002).

[2] P.D. Nellist et al., Science 305, 1741 (2004).

[3] K. van Benthem, Y. Peng and S.J. Pennycook, Mat. Res. Soc. Symp. Proc. 839 (2005). 


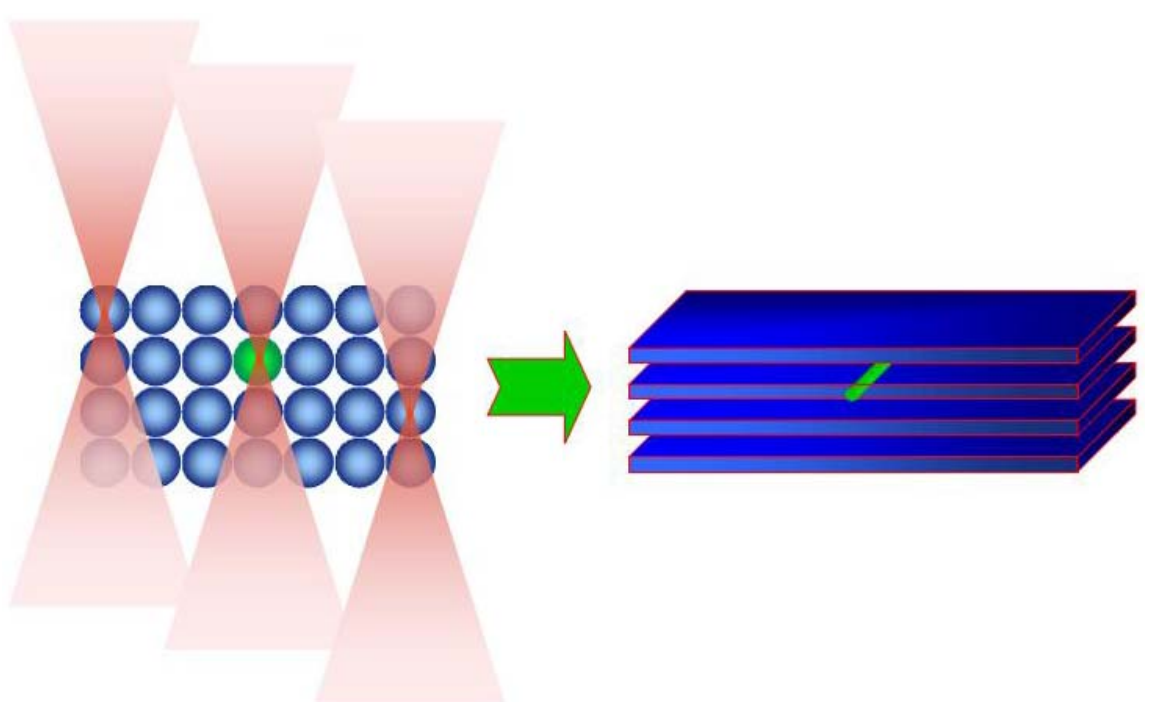

Figure 1: Traditional TEM provides a two-dimensional projection of the true three-dimensional specimen. By consecutively focusing the electron probe into different planes of the specimen, a slice-by-slice image of the three-dimensional structure is available, which is realized by recording through-focal series.
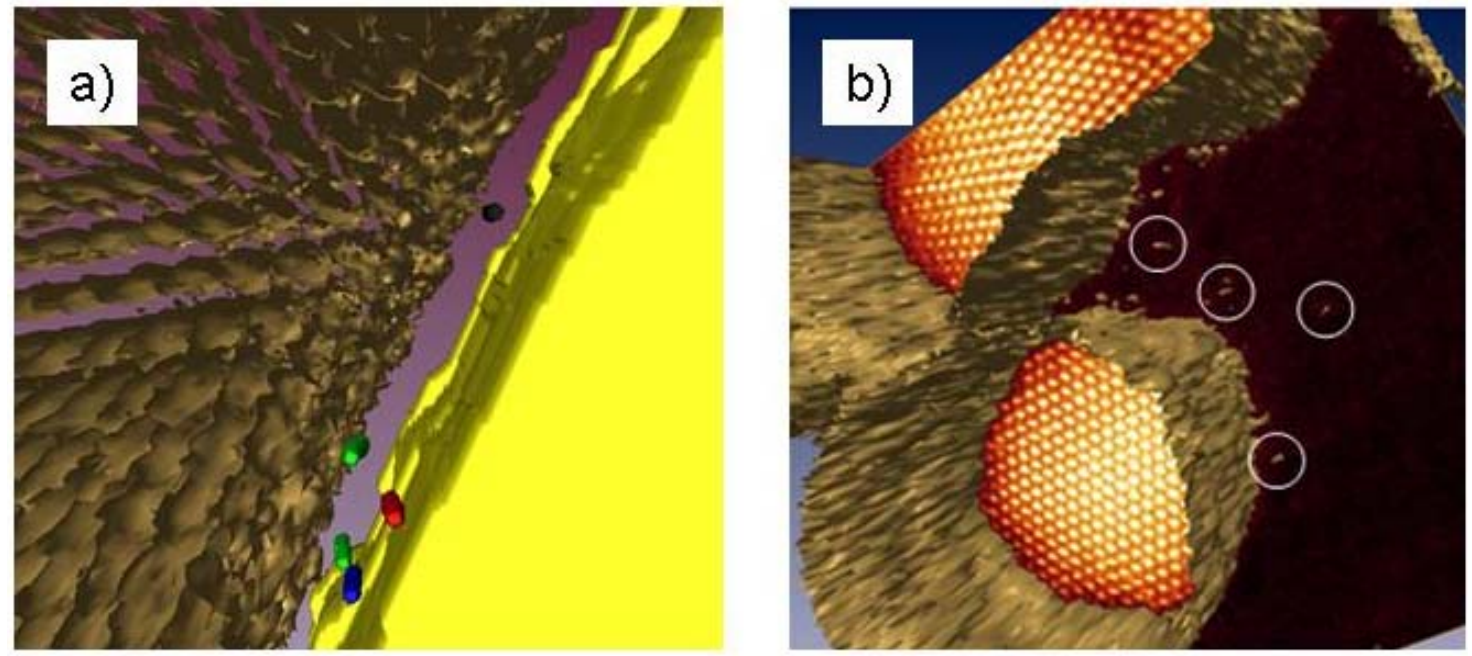

Figure 2: a): Single Hf atoms (marked green, blue, red, and black) are three-dimensionally located within a $\mathrm{SiO}_{2}$ film (invisible in this representation), which is located in between the $\mathrm{Si}$ wafer (gold) and an $\mathrm{HfO}_{2}$ thin film (bold yellow).

b): Reconstructed Au clusters on a carbon support film. The white circles mark single Au atoms present in the vicinity of the Au clusters. 